\title{
Acoustic Emission Source Localization Using Embedded Sensors in Concrete
}

\author{
Lei Qin ${ }^{a}$, Jiapeng Li, Xi Liu, Shidong Sun, Xiaojun Zhu \\ School of Civil Engineering and Architectural Engineering, University of Jinan, Jinan 250022, China \\ ${ }^{a}$ cea_qinl@ujn.edu.cn
}

Keywords: 1-3 transducer, concrete, acoustic emission, accurate location, wave velocity.

\begin{abstract}
This paper described a study on acoustic emission (AE) in the specimens of cement-based materials, especially concrete specimens. 1-3 cement-based piezoelectric composites that have good compatibility and broadband frequency, and they have been developed for health monitoring of concrete structures. It is suitable for 1-3 AE sensor to be embedded in concrete. The wave speed has a significant effect on the $\mathrm{AE}$ location results. The experiments between $\mathrm{AE}$ wave velocity and different distances condition under mortar and concrete two cement-based materials were conducted. The transducers were embedded in concrete, and pencil lead break test was implemented. It can be seen that the transducers embedded in concrete is more accurate and effective when compared with external test results. We could draw a conclusion that it was convenient for an embedded 1-3 AE transducer array to conduct accurate location monitoring of the development of cracks in concrete structure by online and in real-time inexpensively.
\end{abstract}

\section{Introduction}

AE examination is a rapidly maturing non-destructive testing method with demonstrated capabilities for monitoring structural integrity, detecting leaks and incipient failures and for characterizing material behavior [1]. AE technique has been widely used in the field of civil engineering for structural health monitoring (SHM) [2-4]. Considering the number of AE hits and maximum amplitude of AE signal, it is easy to estimate the damage degree in concrete structures. As a result, it is effective to provide sufficient failure warning and life-time assessment at early ages. The 3-D locations of the AE sources can be performed by taking into account arrival time differences of $\mathrm{AE}(\mathrm{TDOA})$ waveforms at each $\mathrm{AE}$ sensor, because the $\mathrm{AE}$ sources localization has great effect on the fracture process assessment and health monitoring of the concrete structures[5,6]. Therefore, it is necessary to obtain accurate and reliable determination of the AE signal arrival time. In the signal analysis, the onset time is usually picked as the point where the first difference between the signal and the noise take place. To improve the accuracy, various algorithms have been proposed for automatic detection of onset time.

In this paper, 1-3cement-based piezoelectric sensor was regarded as $\mathrm{AE}$ transducer to detect $\mathrm{AE}$ wave velocity in different distances condition under various cement-based materials. The characteristics of fracture processes of cement-based materials were investigated under different distances by analyzing the AE parameters. 1-3 AE sensor can be embedded in concrete as a coarse aggregate due to its compatibility with concrete [7]. Moreover, 1-3 AE sensor can also detect AE and transform them into electrical signals. Not only can a data processing device captures the electrical signals, but also it can recognize its waveform characters. Therefore, the data processing device could locate the source position of the elastic wave and trace the crack or damage process of the concrete structure by analyzing the captured information [8].Experiment results show that the localization accuracy was greatly improved. 


\section{Experimental setup}

\subsection{1-3 Cement-based piezoelectric ceramic sensor Section Headings.}

The compatibility between the AE sensor and matrix material is a problem in AE health monitoring. As a result, it is necessary for concrete to find a kind of materials to have relatively broadband frequency response and be suitable to detect signals, then, a type of 1-3 cement-based piezoelectric composites was employed. Up to now, piezoelectric composites with ten types $(0-0,0-1,0-2,0-3,1-1$, $1-2,1-3,2-2,2-3,3-3)$ of connectivity patterns have been fabricated and applied for sensor [9]. In this paper, a type of 1-3 cement-based piezoelectric sensor was used to conduct experiments. The schematic diagram of the composite and the sensor can be shown in Fig. 1.

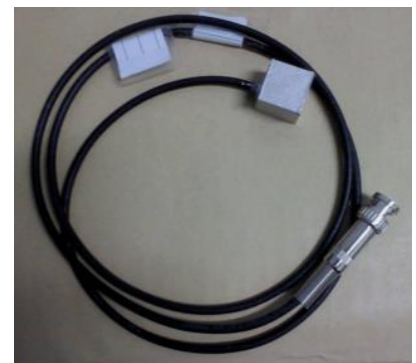

\subsection{Specimen preparation}

Fig. 1. 1-3 cement-based piezoelectric composite sensor.

Four beams made of concrete or mortar were cast in Fig2, all of the beams were $150 \times 150 \times 550 \mathrm{~mm}$. The mixture properties of concrete and mortar are listed in Table 1.

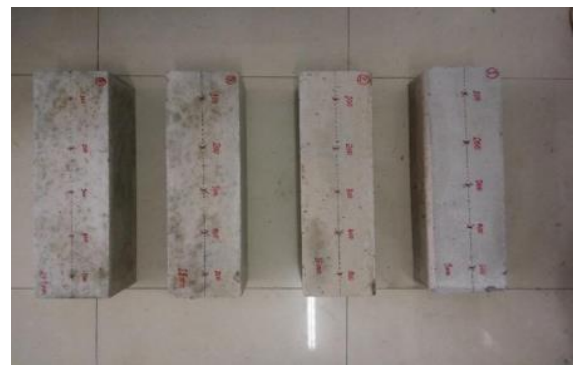

Fig. 2. Fours $150 \times 150 \times 550 \mathrm{~mm}$ beams.

Table 1. The ratio of mixture proportion of the material.

\begin{tabular}{|c|c|c|c|}
\hline cement & sand & aggregate & water \\
\hline 1 & 1.50 & 3.00 & 0.45 \\
\hline 1 & 1,50 & 3.00 & 0.45 \\
\hline 1 & 1.50 & 0 & 0.40 \\
\hline 1 & 1.50 & 0 & 0.40 \\
\hline
\end{tabular}

\subsection{Test procedure}

Pencil lead break testing was implemented to determine the wave velocity can be shown in Fig3.The position of sensor 1 was fixed when measuring the velocity using surface bonded WSa sensor. However, the position of sensor 2 moves with the AE source and the pencil was broken at the side of the sensor. The distance from the AE source to sensor 1 was 10, 20,30 and $40 \mathrm{~cm}$ respectively. Five points were tested for each distance. Fig. 4 show the velocity measurement using surface bonded WSa sensor. In the test, sensor 1 was embedded into concrete specimen. The distance and AE source were same as the above test. Fig.5. showed the velocity measurement using embedded AE sensor. The wave velocity was calculated by using the onset time difference of the two sensors. 


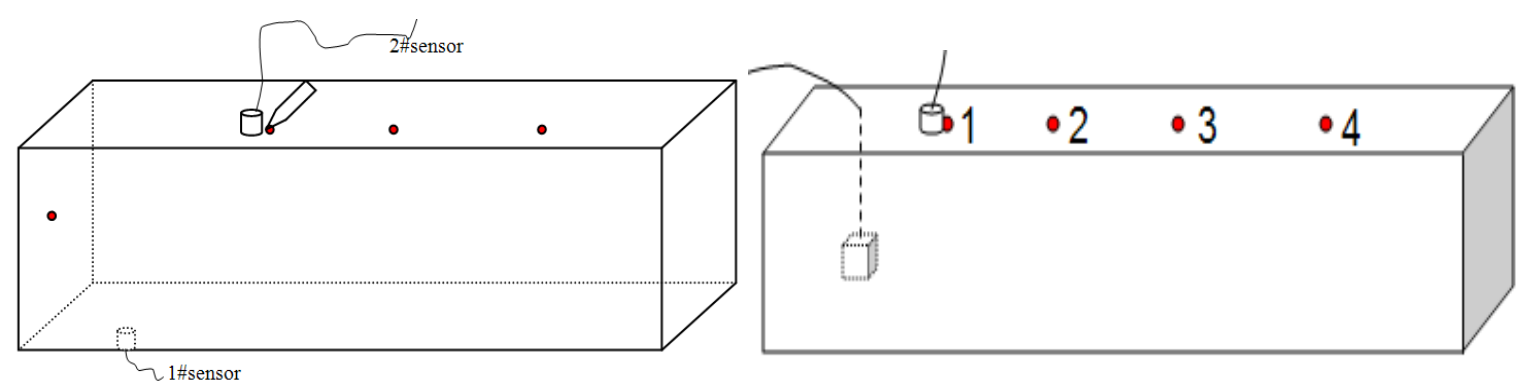

Fig.3. Pencil lead break testing.

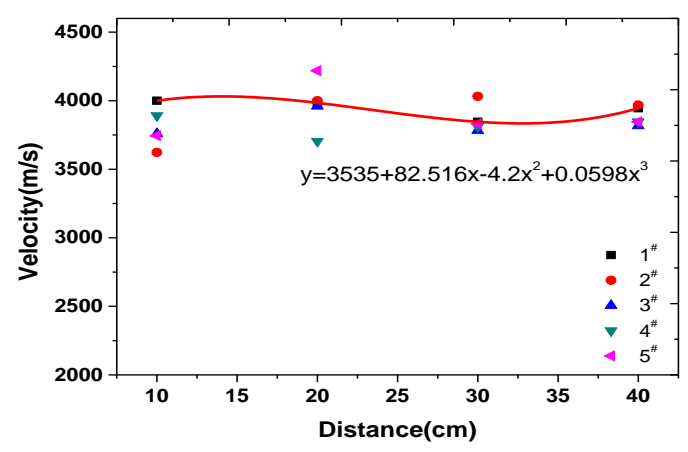

Mortar specimens.

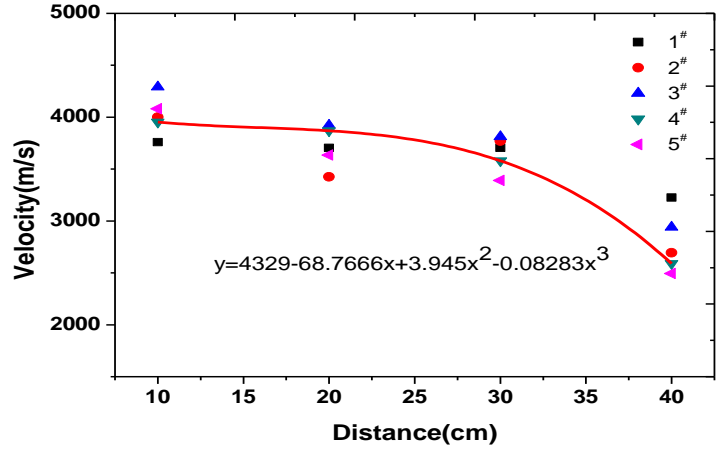

Concrete specimens.

Fig. 4 The measured velocity using surface bonded sensors in mortar and concrete specimens.

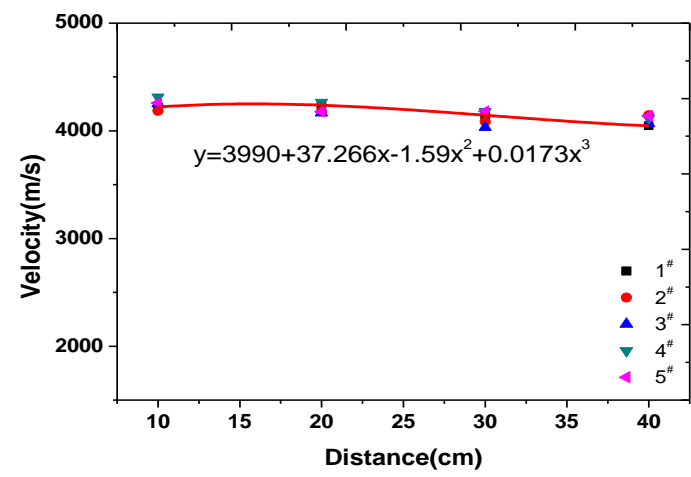

Mortar specimens.

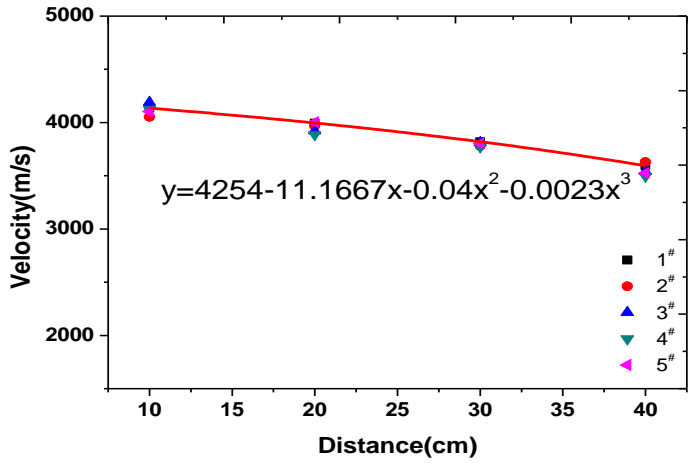

Concrete specimens.

Fig.5. The velocity obtained by the embedded sensors in mortar and concrete specimens.

\section{Results and analysis}

Fig. 4(using surface bonded sensors) shows that with the increasing of the distance, the velocity almost had no change. In concrete, wave velocity decreased obviously with distance. The average velocity was about $4000 \mathrm{~m} / \mathrm{s}$ at $10 \mathrm{~cm}$ and it decreased to about $2500 \mathrm{~m} / \mathrm{s}$ when the distance was $40 \mathrm{~cm}$. It was obviously the inhomogeneity of concrete induced the decreasing of the measured velocity.

Fig. 5(the embedded sensors) showed that with the increasing of the distance, the velocity almost had no change in mortar, and in concrete, the average velocity is about $4000 \mathrm{~m} / \mathrm{s}$ at $10 \mathrm{~cm}$ and it decrease to about $3500 \mathrm{~m} / \mathrm{s}$ when the distance was $40 \mathrm{~cm}$.

Compared with the surface bonded sensors, the velocity of embedded sensors are higher, and the stability of wave velocity is well. Moreover, the decrease of wave velocity is smaller. The reason why embedded sensors conduct well is that 1-3 AE sensor can be embedded in concrete as a coarse aggregate due to its compatibility with concrete, which is beneficial to receiving signal..

\section{Summary}

The results indicated that 1-3 AE sensor can be embedded in concrete as a coarse aggregate due to its compatibility with concrete, which is good for receiving signal [10].And the embedded 1-3 AE 
transducer does well in conducting accurate location monitoring of the development of cracks in concrete structure.

\section{Acknowledgements}

This work was financially supported by the National Natural Science Foundation of China (51378239).

\section{References}

[1] R.K.Miller and Mclntire: Acoustic emission testing Nondestructive Testing Handbook. American Society for Nondestructive Testing.12 (1999) 1-22.

[2] M. Ohtsu and S. Yuyama : Recommended practice for in situ monitoring of concrete structures by acoustic emission. In 15th International acoustic emission symposium. 13 (2000) 263-268.

[3] T. Shiotani : Evaluation of repair effect for deteriorated concrete piers of intake dam using AE activity. Adv. Mater. Res.13 (2014) 175-80.

[4] M. Ohtsu : Simplified moment tensor analysis and unified decomposition of acoustic emission source: application to in situ hydro fracturing test. Constr. Build. Mater. 24 (1998) 1991-96.

[5] B.Q. Dong, Y.Q. Liu, N.X. Han, H.F. Sun, F. Xing, D.D. Qin, Study on the microstructure of cement-based piezoelectric ceramic composites. Constr. Build. Mater. 72 (2014) 133-138.

[6] Z.J. Li, F.M. Li, X.S. Li, W.L. Yang, P-wave arrival determination and AE characterization of concrete. J. Eng. Mech. 126 (2) (2000) 194-200.

[7] M. Ohtsu: Quantitative AE techniques standardized for concrete structures. Adv. Mater. Res. 15 (2006) 183-192.

[8] Y. Lu, Z. Li, L. Qin, Signal-based acoustic emission monitoring on mortar using cement-based piezoelectric sensors. ACI. Mater. J. 108 (2) (2011) 178-186.

[9] Z.J. Li, D. Zhang, K.R. Wu, Cement-based 0-3 piezoelectric composites. J. Am. Ceram. Soc. 85 (2) (2002) 305-313.

[10] A. Behnia ,H.K. Chai ,M. Yorikawa , S. Momoki , M. Terazawa , T. Shiotani : Integrated nondestructive assessment of concrete structures under flexure by acoustic emission and travel time tomography. Constr. Build. Mater. 25 (2) (2011) 167-175. 